\title{
Resistance of Plamodium falciparum to Antimalarial Drugs in Zaragoza (Antioquia, Colombia), 1998
}

\author{
Silvia Blair-Trujillo+, Leidy Lacharme-Lora,Jaime Carmona-Fonseca
}

Grupo Malaria, Facultad de Medicina, Universidad de Antioquia, Carrera 51 D, No. 62-29, Medellín, Colombia

Plasmodium falciparum sensitivity to chloroquine (CHL), amodiaquine (AMO) and sulfadoxine/pyrimethamine (SDX/PYR) was assessed in vivo and in vitro in a representative sample from the population of Zaragoza in El Bajo Cauca region (Antioquia-Colombia).

There were 94 patients with $\mathrm{P}$. falciparum evaluated. For the in vivo test the patients were followed by clinical examination and microscopy, during 7 days. The in vitro test was performed following the recommendations of the World Health Organization. The in vivo prevalence of resistance to CHL was 67\%, to AMO 3\% and to SDX/PYR 9\%.

The in vitro test showed sensitivity to all antimalarials evaluated. Concordance for CHL between the in vivo and in vitro tests was $33 \%$. For AMO and SDX/PYR, the concordance was $100 \%$.

We conclude that a high percentage of patients are resistant to CHL (in vivo). A high rate of intestinal parasitism might explain in part, the differences observed between the in vivo and the in vitro results.

Therefore, new policies and treatment regimens should be proposed for the treatment of the infection in the region. Nationwide studies assessing the degree of resistance are needed.

Key words: Plasmodium falciparum - antimalarial drugs - resistance - Colombia

The first report of chloroquine (CHL) resistant Plasmodium falciparum was made in Colombia in 1961 (Moore \& Lanier 1961). In the following years, other studies showed a high prevalence of resistance in many regions of the country (Walker \& Lopez 1968). However, a detailed review of publications on resistance between 1961 and 1986 concluded that CHL resistance was only $19 \%$ (Blair 1986).

No additional studies were been made until recently when malaria incidence increased in all the country, with a parasite annual index (PAI) from $0.9 \%$ in 1967 , to $10 \%$ in 1998 (Ministerio de Salud de Colombia 1999). Therefore, the issue of resistance to antimalarials has become more relevant. In a study carried out in Chocó (on the Pacific Coast of Colombia), resistance to CHL (47\%) and sulphadoxine-pyrimethamine (SDX/PYR)(6\%) was informed (Osorio et al. 1997). En Turbo, Uraba region, we found CHL resistance in 97\%, amodiaquine (AMO) in 7\% and SDX/PYR in $13 \%$. A lower concordance was observed between in vivo and in vitro tests for CHL, but higher concordance for AMO and SDX/PYR (Blair et al. 2001). Turbo has an intermediate level of endemicity for malaria.

The present study evaluated the in vivo and in vitro sensitivity of $P$. falciparum to CHL, AMO and SDX/PYR in a representative sample taken from the population of Zaragoza (Bajo Cauca region), located in a highly endemic area for malaria and where the incidence of $P$. falciparum

This work was supported by Direccion Seccional de Salud de Antioquia and Universidad de Antioquia.

${ }^{+}$Corresponding author. Fax: +574-2-633509. E-mail: sblair@catios.udea.edu.co

Received 12 July 2001

Accepted 9 October 2001 infection in 1998 was 90.6\%o inhabitants (Ministerio de Salud de Colombia 1998).

\section{MATERIALS AND METHODS}

Patients - A sample of the population of Zaragoza infected by $P$. falciparum was selected applying the following formula (Martínez 1984, 1987, Blair et al. 2001)

$$
\mathrm{n}=\mathrm{N} \mathrm{Z}^{2} \mathrm{p}(1-\mathrm{p}) /\left[\left(\mathrm{N} \mathrm{d}^{2}\right)+\left(\mathrm{Z}^{2} \mathrm{p}(1-\mathrm{p})\right]\right.
$$

where $\mathrm{n}$ is the number of individuals of the sample, $\mathrm{N}$ is the number of $P$. falciparum infected individuals within the population, $\mathrm{Z}$ is the number of units of the standard normal curve which define the confidence interval, $p$ is the prevalence of resistance to the drug, (1-p) is the absence of resistance, $\mathrm{d}$ is the sample error.

In Zaragoza, during 1997, 2,000 cases of P. falciparum malaria were diagnosed. The prevalence (supposed) of resistance to CHL was $50 \%$, to AMO $10 \%$ and to SDX/ PYR $10 \%$. A confidence interval of $95 \%$ was considered $(\mathrm{Z}=1.96)$. Sample errors were 0.18 for the CHL group, and 0.105 for the AMO and SDX/PYR groups. Thirty individuals were included in the CHL group, 31 in the AMO group and 31 in the SDX/PYR group.

Subjects attending the local hospital for diagnosis and treatment were enrolled from February to May 1998. All patients reported fever among other symptoms. Patients were included in the study in a consecutive fashion, according to the time of attendance. Patients with $P$. falciparum malaria were interviewed and evaluated by the researchers and also informed about the purpose of the study. The informed consent was obtained from adults and children's tutors. At this point, random distribution of each individual into one of the groups was carried out. The patient was informed of each of the possible treatments administered but did not know which treatment was receiving. Treatments were administered to each patient by the researchers. 
The sample selected was representative of the population of $P$. falciparum patients attending the local hospital according to age, sex and social-economical conditions.

Patients with $P$. falciparum malaria were included in the study if they were $\geq 1$ year-old, had between 900 and 80,000 parasites $/ \mathrm{ml}$ and were resident of the area (to make possible a 7 days follow-up). Patients were excluded if they had mixed malaria infection, complicated malaria, intense vomiting or diarrhea, or presence of 4-aminoquinolines (Bergqvist et al. 1985) or SDX/PYR (or its metabolites) in urine (Mount et al. 1996).

In vivo sensitivity - In vivo response to the therapy was assessed following clinical and parasitological criteria.

Clinical examination and blood parasite counts were performed on days $0,1,2,3,7$ in all patients. CHL and AMO were administered at $25 \mathrm{mg} / \mathrm{kg}$ during 3 days (10 $\mathrm{mg} / \mathrm{kg}$ on day $0 ; 7.5 \mathrm{mg} / \mathrm{kg}$ on days 1 and 2) and SDX/PYR was given at $25 / 1,25 \mathrm{mg} / \mathrm{kg}$ single dose. Ingestion of treatments was supervised by one of the examiners.

Parasitaemia was measured on days 0,3 and 7 by thick smear and was considered negative when no parasites were found after examination of 100 fields.

Antimalarial response was classified as follows:

\section{Parasitological response}

Parasitological failure: parasitaemia on day 2 higher than on day 0 .

Resistance III ( $R$ III): parasitaemia on day 3 higher or $25 \%$ of parasitaemia on day 0 , or parasitaemia on day $2 \geq 100 \%$ of day 0 .

Resistance II $(R I I)$ : parasitaemia on day $3<25 \%$ of on day 0 , but positive parasitaemia between days 4 and 7 .

Resistance $I(R I)$ : a negative blood smear on day 3 and a positive blood smear on any day between days 7 and 14 (although in this study patients were only followed for 7 days).

Sensitive/Resistance I (S/R I): a negative blood smear, or parasitaemia $<25 \%$ of on day 0 , on day 3 plus a negative blood smear between days 4 and 7 (Barat et al. 1996).

\section{Clinical response}

Early failure $(E F)$ : any of the following situations within the first 3 days of treatment:

Signs of complicated malaria with presence of parasitaemia on days 1, 2 or 3;

Parasitaemia on day $2 \geq 100 \%$ of day 0 or on day $3 \geq$ $25 \%$ of day 0 .

Late failure $(L F)$ : any of the following situations between days 4 and 7 post-treatment.

Clinical relapse or signs of malaria complicated with parasitaemia.

Satisfactory response (SR): when there is no evidence of EF or LF and parasite clearance is confirmed during the period of follow up.

Methodology of the in vitro sensitivity test: in vitro sensitivity of $P$. falciparum to drugs was determined using the standardized the World Health Organization microtechnique (WHO 1990) with an incubation time of $36 \mathrm{~h}$ to promote schizonts maturation. Reagents were purchased from WHO. The test was performed in 8 well microplates with two-fold dilutions of the drug.
Statistical analysis - Data were processed using the Epi info 6.04 program. Treatment groups were randomly assigned and the random distribution of the variables (sex, age and origin) in the three groups was verified by a $\chi^{2}$ test. The Kruskal and Wallis test was used to establish the relation between "response to the treatment" (four values) and "parasite density according to the day of control". Association between "the response to the treatment" (four values) and every variable (sex, age and origin) was estimated for each treatment. The association between the variables "response to the treatment in vivo" (sensitive/resistant) and "response to the treatment in vitro" (sensitive/resistant) was measured with the $\chi^{2}$ Fisher test, since the two tests (in vivo, in vitro) were performed independently.

\section{RESULTS}

All enrolled patients were followed throughout the planned period; $54 \%$ were men and $46 \%$ were women. In the CHL group there was a greater proportion of men $(67 \%)$ and in the AMO and SDX/PYR groups there were similar proportions of men and women. Of 94 patients, 50\% were under 15 years old. When comparing the age in each treatment group, we also found a similar proportion between children and adults. Patients came from urban areas (58\%) and from their rural areas (42\%). A statistically significant association between the treatment given and the variables sex, age and place of origin was not found (age: $\chi^{2}=7.16$, $\mathrm{df}=4, \mathrm{p}=0.127885$; sex: $\chi^{2}=2.98, \mathrm{df}=2, \mathrm{p}=0.225480$; origin: $\chi^{2}=5.12$, df $=2, \mathrm{p}=0.225849$ ). This result indicates the random selection of the patients.

A total of $7 \%$ of patients had prophylaxis history; $40 \%$ of them used medicinal plants (Exotic Muraya L. Momordica charantia, Gliricidia sepium) and $30 \%$ received CHL at least once; $6 \%$ had history of antimalarial drugs intake during the last 20 days, but when the levels of the drugs in urine were tested, this could not be confirmed.

A total of $74 \%$ of patients given $\mathrm{CHL}, 64 \%$ given AMO and $88 \%$ given SDX/PYR showed an initial parasite density under 10,000 parasites/ $\mu$ l. The initial mean parasite density of patients given CHL or AMO was very similar. A statistically significant difference between the three mean values (Kruskal \& Wallis $=5.088 ; \mathrm{df}=2 ; \mathrm{p}=0.078538$ ) was not found. Following of parasitaemia values in each group clearly shows that patients treated with CHL presented a daily parasite density higher than other groups (Figure).

In vivo $P$. falciparum response to $C H L$ - Of the 30 patients studied, $14(47 \%)$ had resistance to CHL and there was a parasitological failure in $20 \%$. Since parasitological failure is an expression of resistance, that was explored separately, the total resistance to $\mathrm{CHL}$ was $67 \%$ (Table I).

Table II shows the results of the in vivo test, the mean of daily parasite density and parasitological response to CHL. A statistically significant difference was not found; $67 \%$ of patients from this group were men and $33 \%$ were women; the mean age was 20 years old; $73 \%$ lived in the urban area and $23 \%$ in the rural area.

In vivo sensitive and resistant strains were similar by sex, age and origin, as evident by the lack of association 


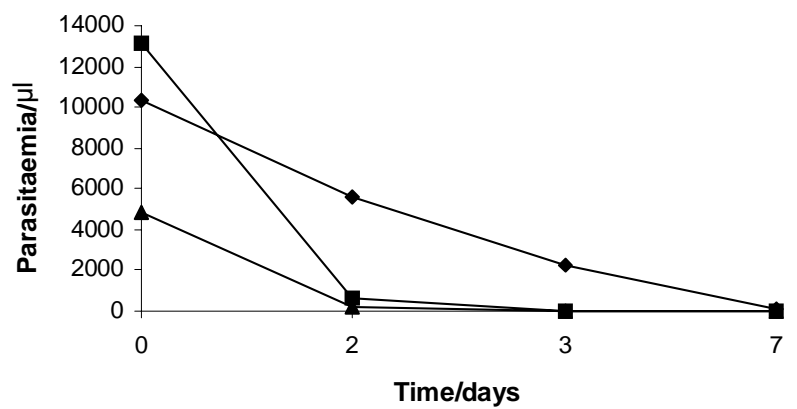

$\multimap$ Chloroquine $\multimap-$ Amodiaquine $\longleftarrow$ Sulfadoxine/pyrimethamine

Daily mean Plasmodium falciparum parasitaemia of the three experimental groups

between these variables and response to treatment (age: $\chi^{2}=1.76, \mathrm{df}=6, \mathrm{p}=0.53276$; sex: $\chi^{2}=2.63, \mathrm{df}=3, \mathrm{p}=$ 0.45312 ; origin: $\chi^{2}=6.73, \mathrm{df}=3, \mathrm{p}=0.1637$ ). This lack of association between the variables confirms that random assignation to each treatment group was successful.

In vivo $P$. falciparum response to $A M O$ - Of 31 patients, $30(97 \%$ ) were sensitive to AMO and only $3 \%$ (one patient) exhibited RII. Based on the initial parasite density, a statistically significant difference between the sensitive patients and the resistant one was not found (Kruskal \& Wallis $=0.612 ; \mathrm{df}=1 ; \mathrm{p}=0.433848)$ (Table III). Initial parasite density is not distributed as a normal line;
$51 \%$ of the patients from this group were men and $49 \%$ women; the mean age was 20 years old; $55 \%$ was from the urban area and $45 \%$ from the rural area. A 13-year-old boy from the urban area had resistance to AMO.

In vivo sensitive and resistant strains were similar by sex, age and origin (age: $\chi^{2}=1.88, \mathrm{df}=2, \mathrm{p}=0.39086465$; sex: $\chi^{2}=1, \mathrm{df}=1, \mathrm{p}=1.00$; origin: $\chi^{2}=1, \mathrm{df}=1, \mathrm{p}=1.0$ ). As in the CHL group, this lack of association of the variables confirm the success of the random selection .

In vivo P. falciparum response to SDX/PYR - Thirty (91\%) patients of 33 were sensitive to SDX/PYR; 6\% exhibited RII and 3\% parasitological failure. Mean initial parasite density was the same in all of them, but there were differences during the $2 \mathrm{nd}, 3 \mathrm{rd}$ and 7 th days (see Table IV). Initial parasite density was not distributed as a normal line; $45 \%$ of the patients from this group were men and $55 \%$ women; the mean age was 20 years old; $45 \%$ was from the urban area and $55 \%$ from the rural area. Sensitive and resistant patients were similar according to the variables "sex, age and origin" (age: $\chi^{2}=1.81, \mathrm{df}=4, \mathrm{p}=$ 0.76810113 ; sex: $\chi^{2}=1.27, \mathrm{df}=2, \mathrm{p}=0.52964116$; origin: $\left.\chi^{2}=3.96, \mathrm{df}=2, \mathrm{p}=0.13806924\right)$

In vitro $P$. falciparum response - Table $\mathrm{V}$ shows the number of cultures and their response to the drug. When evaluating the growth rate of schizonts in the wells with the different drugs tested, it was concluded that all the cultures were sensitive. It is very important to show clearly that in all the cultures the mean schizonts growth rate was greater than $10 \%$ of the asexual parasite density (criteria

TABLE I

In vivo Plasmodium falciparum response to the different antimalarials

\begin{tabular}{|c|c|c|c|c|c|c|c|c|}
\hline \multirow{2}{*}{$\begin{array}{l}\text { Sample } \\
\mathrm{CHL}=30\end{array}$} & \multirow{2}{*}{$\begin{array}{l}\text { RIII } \\
2(6.7 \%)\end{array}$} & \multirow{2}{*}{$\frac{\text { RII }}{12(40 \%)}$} & \multirow{2}{*}{$\frac{\mathrm{RI}}{0}$} & \multicolumn{2}{|c|}{ Resistance (R) } & \multirow{2}{*}{$\begin{array}{c}\text { Failure } \\
6 / 20 \%\end{array}$} & \multirow{2}{*}{$\begin{array}{c}\text { Sensitivity } \\
10(33 \%)\end{array}$} & \multirow{2}{*}{$\begin{array}{c}\text { Overall R } \\
67 \%\end{array}$} \\
\hline & & & & 14 & $47 \%$ & & & \\
\hline $\mathrm{AMO}=31$ & 0 & $1(3 \%)$ & 0 & 1 & $3 \%$ & 0 & $30(97 \%)$ & $3 \%$ \\
\hline $\begin{array}{l}\text { SDX/ } \\
\text { PYR = } 33\end{array}$ & 0 & $2(6 \%)$ & 0 & 2 & $6 \%$ & $1 / 3 \%$ & $30(91 \%)$ & $9 \%$ \\
\hline
\end{tabular}

CHL: chloroquine; AMO: amodiaquine; SDX/PYR: sulphadoxine-pyrimethamine

TABLE II

In vivo response and parasitaemia to chloroquine in Plasmodium falciparum patients

\begin{tabular}{|c|c|c|c|c|c|c|}
\hline \multirow[t]{2}{*}{ Response } & \multicolumn{2}{|c|}{ Patients } & \multicolumn{4}{|c|}{ Mean parasitaemia ${ }^{b}$} \\
\hline & No. & $\%$ & Day 0 & Day 2 & Day 3 & Day 7 \\
\hline Sensitive & 10 & 33.3 & 10217 & 1040 & 151 & 0 \\
\hline Resistance II & 12 & 40.0 & 10004 & 3524 & 811 & 234 \\
\hline Resistance III & $2^{a}$ & 6.7 & 30395 & 13351 & 20198 & N.A. \\
\hline Parasitological failure & 6 & 20 & 4529 & 14644 & N.A. & N.A. \\
\hline
\end{tabular}

N.A: not available; $a$ : two patients had 1,490 and 59,300 parasites $/ \mu \mathrm{ml} ; b$ : no statistically significant differences were observed between the results obtained on day 0 when compared with results of any other day $(\mathrm{H}$ Kruskal-Wallis $=0,567 ; \mathrm{df}=3 ; \mathrm{p}=$ 0,904019), but significance was observed when comparing mean results of from days 2, 3 and 7 :

\begin{tabular}{lccc} 
Day & H Kruskal-Wallis & df & p value \\
\hline 2 & 12.784 & 3 & 0.005127 \\
3 & 14.508 & 3 & 0.002289 \\
7 & 12.224 & 3 & 0.000472 \\
\hline
\end{tabular}


TABLE III

In vivo response and parasitaemia to amodiaquine of Plasmodium falciparum infected patients

\begin{tabular}{|c|c|c|c|c|c|c|}
\hline \multirow[t]{2}{*}{ Response } & \multicolumn{2}{|c|}{ Patients } & \multicolumn{4}{|c|}{ Mean parasitaemia/ $\mu l$} \\
\hline & No. & $\%$ & Day 0 & Day 2 & Day 3 & Day 7 \\
\hline Sensitive & 30 & 97 & 13167 & 713 & 16.7 & 0 \\
\hline Resistance II & 1 & 3 & 11656 & 80 & 5 & 3 \\
\hline Total & 31 & 100 & & & & \\
\hline
\end{tabular}

No statistically significant differences were observed between the results obtained on day 0 when compared with results of any other day (H Kruskal- Wallis $=0.612 ; \mathrm{df}=1 ; \mathrm{p}=0.433848)$, similarly no significance was observed between days 2 and 3 , but significance was observed when comparing mean results of day 7 :

\begin{tabular}{lccc} 
Day & H Kruskal Wallis & df & p value \\
\hline 2 & 0.003 & 1 & 0.955344 \\
3 & 2.355 & 1 & 0.1249087 \\
7 & 30.00 & 1 & 0.000000 \\
\hline
\end{tabular}

TABLE IV

In vivo response and parasitaemia to sulfadoxine/pyrimethamine in Plasmodium falciparum patients

\begin{tabular}{|c|c|c|c|c|c|c|}
\hline \multirow[t]{2}{*}{ Response } & \multicolumn{2}{|c|}{ Patients } & \multicolumn{4}{|c|}{ Mean parasitaemia/ $\mu 1^{a}$} \\
\hline & No. & $\%$ & Day 0 & Day 2 & Day 3 & Day 7 \\
\hline Sensitive & 30 & 90.9 & 4852 & 67 & 14 & 0 \\
\hline Resistance II & 2 & 6.1 & 5432 & 210 & 385 & 104 \\
\hline Parasitological failure & 1 & 3 & 3920 & 4831 & N.A. & N.A. \\
\hline Total & 33 & 100 & & & & \\
\hline
\end{tabular}

N.A: not available; $a$ : no statistically significant differences were observed between the results obtained on day 0 when compared with results of any other day (H Kruskal- Wallis $=0,480 ; \mathrm{df}=2 ; \mathrm{p}=0,786824$ ), but significance was observed when comparing mean results of from days 2,3 and 7:

\begin{tabular}{llll} 
Day & H Kruskal-Wallis & df & p value \\
\hline 2 & 6.094 & 2 & 0.047501 \\
3 & 11.058 & 2 & 0.000883 \\
7 & 30.968 & 2 & 0.000000 \\
\hline
\end{tabular}

TABLE V

In vitro sensitivity to chloroquine, amodiaquine y sulfadoxine/ pyrimethamine

\begin{tabular}{lcc}
\hline Samples Cloroquine Amodiaquine & $\begin{array}{c}\text { Sulfadoxine/ } \\
\text { pyrimethamine }\end{array}$ \\
\hline
\end{tabular}

\begin{tabular}{lccc} 
Plated & 30 & 31 & 33 \\
Successfully & 18 & 16 & 12 \\
$\quad$ cultured & & 16 & 12 \\
Sensitive & 18 & 0 & 0 \\
Resistant & 0 & 16 \\
\hline
\end{tabular}

used by the WHO to evaluate the quality of the in vitro test) with low concentration of drug. In the case of the in vitro SDX/PYR response, inhibitory concentration 50 was found in well $\mathrm{B}(10 / 0.125 \mathrm{pmol})$ and inhibitory concentration 90 in well C (30/0.375 pmol).

Concordance between in vivo and in vitro responses - Table VI shows the co-relation between in vivo and in vitro results. The in vitro results only include the cases of successful culture. However, there were few cases with unknown result.
It must be noticed that in all in vivo and in vitro sensitivity tests a concordance of $100 \%$ was observed in both AMO [(15+0)/15] and SDX/PYR [(9+0)/9] cultures. For CHL, the concordance between both tests was only of $33 \%[(6+0) / 18]$.

\section{DISCUSSION}

Low frequency (3-9\%) of in vivo resistance was observed to AMO and SDX/PYR, 100\% of concordance was seen between the in vivo and in vitro tests fro these drugs. On the other hand in vivo resistance to CHL was very high (67\%) and concordance was (33\%). Such high levels of resistance have been registered in many malarial zones of the world such as Papua New Guinea, 85\% (Al Yaman et al. 1996); Tanzania, 44\% (Ekvall et al. 1998) and Burundi Highlands, 77.8\% (Di Perri et al. 1998). This resistance has frequently been observed after massive intake of antimalarial medicaments for prophylaxis (WHO 1996); but this was not the case in our study. Two aspects are very remarkable in the zone studied: (a) the high incidence of malaria caused by $P$. vivax and the high frequency of treatment with CHL; (b) this consequently exerts pharmacological pressure on the population and allows some strains of $P$. falciparum to become resistant. In Turbo during the 
TABLE VI

Concordance of in vivo and in vitro response of Plasmodium falciparum to chloroquine (CHL), amodiaquine (AMO) and to sulfadoxine/pyrimethamine (SDX/PYR)

\begin{tabular}{|c|c|c|c|c|c|c|}
\hline \multirow[t]{2}{*}{ Drug } & \multirow{2}{*}{$\begin{array}{l}\text { In vivo } \\
\text { response }\end{array}$} & \multicolumn{2}{|c|}{ In vitro response } & \multicolumn{2}{|c|}{$\%$ resistance $^{b}$} & \multirow[t]{2}{*}{$\%$ concordance $^{b}$} \\
\hline & & Sensitive & Resistant ${ }^{a}$ & In vivo & In vitro & \\
\hline \multirow[t]{2}{*}{ CHL } & Sensitive & 6 & 0 & 67 & 0 & 33 \\
\hline & Resistant & 12 & 0 & & & \\
\hline \multirow[t]{2}{*}{ AMO } & Sensitive & 15 & 0 & 97 & 0 & 100 \\
\hline & Resistant & 0 & 0 & & & \\
\hline \multirow[t]{2}{*}{ SDX/PYR } & Sensitive & 9 & 0 & 91 & 0 & 100 \\
\hline & Resistant & 0 & 0 & & & \\
\hline
\end{tabular}

$a$ : in vitro, tests with results known were sensitive, however, we did not know the in vitro response of many cases that in vivo analysis were sensitive or resistant:

\begin{tabular}{lcc} 
& Sensitive & Resistant \\
\hline CHL & 4 & 8 \\
AMO & 15 & 1 \\
SDX/PYR & 21 & 3 \\
\hline
\end{tabular}

$b$ : it refers to all cases with results known in both in vivo and in vitro analysis.

same time of the year of the present study, we found in vivo resistance to $\mathrm{CHL}$ of $97 \%$. Both studies were designed in a similar manner and identical measures were taken to avoid loss of patients and guarantee right intake of the drugs in order to avoid bias (Blair et al. 2001). Lack of compliance and presence of vomit or diarrhea were not responsible for the differences observed between the in vivo and the in vitro results since patients were carefully monitored. Similarly, quality of the CHL suministered by the Ministery of Health was checked in three separate laboratories (two in Colombia and one in Uruguay).

WHO states that evaluation of sensitivity of $P$. falciparum to antimalarials is better assessed by in vivo tests, since they reflect better the biological interaction with the drug and the host's response. However, in vivo tests are affected by variables that might be out of control such as absorption deficit due to the diet (Tulpule \& Krishanaswamy 1982, 1983, McElnay et al. 1982, Romero et al. 1993) or other drugs (Mahmoud et al. 1994), increased degradation of the drug into inactive metabolites and presence of intestinal parasite infections. We found prevalence of intestinal parasite infections in all age groups and rural and urban areas in around $50 \%$ of individuals (unpublished data). This might explain in part, the differences observed between the in vivo and the in vitro results and future studies should address this problem.

Explanation for such a high resistance to CHL (67\%) can not be simple. We found that from 18 strains known as sensitive in vitro, 12 were resistant in vivo and 6 sensitive in vivo. Lack of concordance between the in vivo and in vitro tests for CHL have also been reported by other authors (Testa et al. 1998); however, contrary to our findings, more resistance in vitro than in vivo has been generally observed. The low concordance in the CHL groups was also observed in a study carried out in the population of Turbo, where concordance was $23 \%$ for CHL, $82 \%$ for AMO and $100 \%$ for SDX/PYR (Blair et al. 2001).
These results suggest that, similarly to previous observations made in Turbo (Blair et al. 2001), in Zaragoza, Bajo Cauca region (Colombia), there is a high prevalence of CHL resistant $P$. falciparum. We suggest sanitary authorities should study and implement new treatment policies in cases of $P$. falciparum diagnosed in the region. Furthermore, resistance studies in the rest of the country should be carried out to evaluate sensibility to the antimalarials in cases of $P$. falciparum and $P$. vivax.

\section{ACKNOWLEDGEMENTS}

To employees from the Hospital San Rafael in Zaragoza for their collaboration.

\section{REFERENCES}

Al Yaman F, Genton B, Mokela D, Narara A, Raiko A, Alpers MP 1996. Resistance of Plasmodium falciparum malaria to amodiaquine, chloroquine and quinine in the Madang Province of Papua New Guinea. PNG Med J 39: 16-22.

Barat L, Mulenga M, Kapelwa W, Nkunika S, Himonga B, Ettling M, Ruebush T, Bloland P 1996. A standard protocol for assessing and monitoring malaria therapy efficacy in Zambia, Center for Diseases Control and Prevention, Atlanta, Georgia, USA.

Bergqvist Y, Christier H, Funding L, Suther A 1985. Determination of chloroquine and its metabolites in urine: a field method based on ion-pair extraction. Bull WHO 63: 893-898.

Blair S 1986. Resistencia de P. falciparum a drogas en Colombia 1961-1986. Biomedica 6: 95-100.

Blair S, Lacaharme LL, Carmona-Fonseca J, Tobon A 2001. Resistencia de $P$. falciparum a tres fármacos antimaláricos en Turbo (Antioquia, Colombia), 1998. Rev Panam Salud Publica 9: 23-29.

Di Perri G, Olliaro P, Nardi S, Deganello R, Allegranzi B, Bonora S, Vento S, Concia E 1998. Response of uncomplicated falciparum malaria to oral chloroquine and quinine in Burundi Highlands. Acta Trop 70: 25-33.

Ekvall H, Premji Z, Bjorkman A 1998. Chloroquine treatment for uncomplicated childhood malaria in an area with drug resistance: early treatment failure aggravates anaemia. Trans 
$R$ Soc Trop Med Hyg 92: 556-560.

Mahmoud BM, Ali HM, Homeida MM, Bennett JL 1994. Significant reduction in chloroquine biolavailability following coadministration with the sudanese beverages Aradaib, Karkadi and Lemon. J Antimicrob Chemother 33: 1005-1009.

Martínez C 1984. Muestreo, Ecoe, Bogotá, p. 45-47.

Martínez C 1987. Estadística, 4 ed., Ecoe, Bogotá, p. 599-604.

Mc Elnay JC, Mukhtar HA, D'arcy PF, Temple DJ 1982. In vitro experiments on chloroquine and pyrimethamine absorption in the presence of antiacid constituents on Kaolin. J Trop Med Hyg 85: 153-158.

Ministerio de Salud de Colombia 1998. Guía Clínica para el Diagnóstico y Tratamiento de la Malaria en Colombia, Dirección General de Promoción y Prevención, Santa Fe de Bogotá.

Ministerio de Salud de Colombia 1999. Malaria por Departamentos, Muestras Examinadas, Positivas y por Especie en Colombia, Unidad Administrativa de Campañas Directas, Santa Fe de Bogotá.

Moore DV, Lanier SE 1961. Observations of two Plasmodium falciparum infections with an abnormal response to chloroquine. Am J Trop Med Hyg 10: 5-9.

Mount DL, Green MD, Zucker JR, Were JBO, Todd GD 1996. Field detection of sulfonamides in urine the development of a new and sensitive test. Am J Trop Med Hyg 55: 250-253.

Osorio LE, Giraldo LE, Grajales LF, Barat LM, Cordoba F, Arriaga AL, Andrade AL, Ruebush T 1997. Evaluación in vivo de la resistencia de Plasmodium falciparum a cloroquina y sulfa/pirimetamina en Quibdó-Chocó. Biomédica 17 (Supl. 2): 201-202.

Romero A, Matos C, Gonzalez MM, Nuñez N, Bermudez L, De Castro G 1993. Alteraciones de la mucosa gastrica en malaria aguda. GEN 47: 123-128.

Testa J, Traore LK, Nabalma S, Sondo B, Guissou IP 1998. Chloroquine resistance of Plasmodium falciparum. Study of a surveillance method based on placental apposition and determination of blood chloroquine in pregnant women. Sante 8: 293-296.

Tulpule A, Krishanaswamy K 1982. Effect of food on bioavailability of chloroquine. Eur J Clin Pharmacol 23: 271-273.

Tulpule A, Krishanaswamy K 1983. Effect of rice diet on chloroquine bioavailability. Drug Nutr Interact 2: 83-86.

Walker M, López F 1968. Response to drugs of South American strains of $P$. falciparum. Trans $R$ Soc Trop Med Hyg 62: 654-667.

WHO-World Health Organization 1990. In vitro test (mark II) for the assessment of the response of Plasmodium falciparum to chloroquine, mefloquine, quinine, sulfadoxine/ pyrimethamine and amodiaquine. World Health Organization document MAP/87.2 revision 1.

WHO-World Health Organization 1996. Assessment of therapeutic efficacy of antimalarial drugs for uncomplicated falciparum malaria in areas with intense transmission. World Health Organization WHO/MAL/96.107. 